\title{
Postinflationary scalar tensor cosmology and inflationary parameters
}

\author{
Anshuman Maharana ${ }^{1, *}$ and Ivonne Zavala ${ }^{2, \dagger}$ \\ ${ }^{1}$ Harish Chandra Research Institute, HBNI, Chattnag Road, Jhunsi, Allahabad 211019, India \\ ${ }^{2}$ Department of Physics, Swansea University, Singleton Park, Swansea SA2 8PP, United Kingdom
}

(Received 6 February 2018; published 13 June 2018)

\begin{abstract}
Scalar fields provide attractive modifications of pre-BBN cosmology, which have interesting implications for dark matter abundances. We analyze the effect of these modifications on the number of $e$-foldings between the horizon exit of cosmic microwave background modes and the end of inflation $\left(N_{k}\right)$, and examine the consequences for inflationary predictions of various models. We find significant effects in the predictions in the $\left(n_{s}, r\right)$ plane. For a large part of the parameter space, the shift in $N_{k}$ is positive; this ameliorates the tension of $m^{2} \varphi^{2}$ and natural inflation with data.
\end{abstract}

DOI: 10.1103/PhysRevD.97.123518

\section{INTRODUCTION}

Cosmological observations are the source of some of the biggest challenges in physics today. Only 5\% of the energy density of the Universe is baryonic matter, the remaining is dark matter (27\%) and dark energy (68\%). The cosmic microwave background (CMB) and galaxy distributions exhibit correlations even at superhorizon scales, with distinct patterns in the inhomogeneities. The $\Lambda$ CDM model together with an inflationary epoch in the early Universe has become the standard framework used to address these observations.

In this setting, a popular scenario to address dark matter abundance is the weakly interacting massive particle (WIMP) paradigm. At early times, dark matter was in thermal equilibrium with the standard model degrees of freedom (d.o.f.). As the temperature of the Universe decreased, the dark matter interaction rate fell down and its number density became a constant in the comoving frame- the present dark matter abundance is this relic. The required value of the dark matter abundance is obtained for $\left\langle\sigma_{\text {th }} v_{\text {rel }}\right\rangle \simeq 3 \times 10^{-26} \mathrm{~cm}^{3} \mathrm{sec}^{-1}$, where $v_{\text {rel }}$ is the Moller velocity and a thermal average is taken. This gives the typical value of the cross section to be that associated with the weak scale. The emergence of the weak scale from the relic abundance calculation is often cited as the WIMP miracle. Experimentally, there are intense efforts to probe dark matter cross sections [1,2] while future experiments

\footnotetext{
*anshumanmaharana@hri.res.in

e.i.zavalacarrasco@swansea.ac.uk
}

Published by the American Physical Society under the terms of the Creative Commons Attribution 4.0 International license. Further distribution of this work must maintain attribution to the author(s) and the published article's title, journal citation, and DOI. Funded by SCOAP. will further probe the annihilation rate for a wide range of dark matter masses $[3,4]{ }^{1}$

Given the ongoing experimental efforts, the time is ripe to critically examine the inputs that enter into the standard relic abundance calculation and explore alternatives where dark matter is a thermal relic, yet the value of $\left\langle\sigma_{\text {th }} v_{\text {rel }}\right\rangle$ is different from the above quoted value. Of course, it could well be that dark matter is nonthermal. Both avenues have received considerable attention (see, e.g., [6-23]).

A key input for the standard computation of thermal dark matter abundance is that the energy density of the Universe was dominated by radiation at the time that dark matter decoupled. One of the major successes of the hot big bang model is nucleosynthesis (BBN); this requires that the Universe was radiation dominated at the time of production of the light nuclei, the corresponding temperature being $T_{\mathrm{BBN}} \simeq 1 \mathrm{MeV}$ (see $[24,25]$ for the related constraints on late time entropy production). But the history of the Universe prior to $\mathrm{BBN}$ remains highly unconstrained. A modification of the history of the Universe prior to nucleosynthesis, in particular a modified expansion rate of the Universe, can significantly alter the dark matter relic density computations.

Scalar fields with nontrivial evolution provide well motivated modifications of the pre-BBN history with interesting implications for dark matter relic abundances. The simplest scenario with a modified cosmological history is when an extra scalar species is present [26], which does not couple directly to the Standard Model (SM) d.o.f. or dark matter. The energy density of the decoupled scalar dilutes as $\rho_{\phi} \propto a^{-(4+n)}$ with $n>0$ [where $a(t)$ is the scale factor]. This form implies that the scalar energy density dominates over radiation at early times. This in turn leads to

\footnotetext{
${ }^{1}$ For a complete list of direct and indirect current and future experiments on dark matter searches, see [5].
} 
a modification in the relic abundance calculations. Even this simple model leads to novel features in the phenomenology_dark matter annihilation processes can continue to take place long after the decoupling of dark matter from the thermal bath. This was dubbed as relentless dark matter, as it can be thought of as dark matter trying to get back into thermal equilibrium.

More generally, scalar-tensor (ST) theories of gravity arise in extensions of the standard models of cosmology and particle physics. For example, in higher dimensional models, additional scalar fields arise through the compactification of the extra dimensions and couple to the metric with gravitational strength. Thus, the gravitational interaction is mediated by both the metric and scalar fields so that scalar-tensor gravity models represent a departure from standard general relativity (GR). In string theory models of particle physics and cosmology, new ingredients such as D-branes can also appear. For such D-branes, the longitudinal fluctuations are identified with the matter fields such as the SM and DM particles, while transverse fluctuations correspond to scalar fields. These scalars couple conformally and disformally to the matter living on the brane and thus, can modify the cosmological expansion rate felt by matter and hence, the standard predictions for the dark matter relic abundance ${ }^{2}$ [39-41]. An attractive feature of the ST case is the possibility of tracker solutions of the scalar field, which ensure that the scalar field becomes inactive at the onset of BBN (and thereafter) as is required for its successes.

One of the major successes of the inflationary paradigm is the explanation for the approximately scale invariant spectrum of the CMB. Given a model of inflation, the predictions for the scalar spectral tilt $\left(n_{s}\right)$ and the tensor to scalar ratio $(r)$ are determined by the number of $e$ foldings, $N_{k}$, between horizon exit of the CMB modes and the end of inflation. Typically, $N_{k}$ is taken to be in the range 50 to 60 . It is important to keep in mind the inputs that go into deriving this. The derivation makes use of two aspects of the physics of inflation: the condition for horizon exit of the CMB modes $\left(k=a_{k} H_{k}\right.$; where $k$ is the wave number of the CMB modes, $a_{k}$ and $H_{k}$ are the scale factor and Hubble constant at the time of horizon exit) and the relationship between the strength of the scalar perturbations and the energy density at the time of horizon exit. Taking these inputs, the evolution of the energy density from the time of horizon exit to the present epoch and entropy evolution from the end of reheating to the present epoch are tracked. For the evolution, the postinflationary history is taken to be the standard history associated with the hot big bang model. This tracking then yields the equation that determines $N_{k}$,

\footnotetext{
${ }^{2}$ Further studies of the modifications to the relic abundances in conformally coupled scalar-tensor theories have been discussed in [27-38].
}

$$
N_{k} \approx 57+\frac{1}{4} \ln r-\frac{1}{4}\left(1-3 w_{\mathrm{re}}\right) N_{\mathrm{re}},
$$

where $r$ is the tensor to scalar ratio and $w_{\text {re }}, N_{\text {re }}$ are the (effective) equation of state parameter and number of $e$ foldings during the reheating epoch. In the canonical reheating scenario, $w_{\mathrm{re}} \simeq 0$, but more general cases can give $1 / 3<w_{\text {re }}<0[42,43]$. Given the uncertainties associated with reheating and the fact that the tensor mode has still not been detected, Eq. (1) does not fix the value of $N_{k}$ exactly, but under the assumption that the reheating epoch is not highly extended, it serves as the motivation for the usual range of 50 to 60 for $N_{k}$. The input that the postinflationary history is the standard history associated with the hot big bang model is central to the derivation of (1); any modification of the postinflationary history has an effect on the preferred range for $N_{k}$. As we enter the era of precision cosmology, ${ }^{3}$ it is very important to determine $N_{k}$ accurately so that theoretical predictions to confront the data can be made. The models discussed above in the context of dark matter abundance have a nonstandard cosmological history prior to BBN. The goal of this paper is to determine $N_{k}$ for these models and obtain the implications for inflationary predictions.

The rest of the paper is organized as follows. We review theories with scalars in pre-BBN cosmology motivated by dark matter considerations in Sec. II. In Sec. III, we obtain the expressions for $N_{k}$ in these theories, and in Sec. IV, we discuss the implications of our results for the predictions in the $\left(n_{s}, r\right)$ plane of various inflationary models. We conclude in Sec. V.

\section{SCALARS IN PRE-BBN COSMOLOGY}

\section{A. Extra scalar species}

The simplest scenario to modify the cosmological history before the onset of $\mathrm{BBN}$, is to consider a new scalar species $\phi$, which does not couple to matter, and has a nontrivial cosmological evolution in the pre-BBN history. The defining property of a such decoupled scalar is that its energy momentum tensor is conserved separately: $\nabla^{\mu} T_{\mu \nu}^{\phi}=0$. In addition, various components of matter have individually conserved stress tensors $\nabla^{\mu} T_{\mu \nu}^{i}=0$, where the index $i$ runs over the components of matter whose stress tensors are conserved. The consequence of these conservation equations is that the various components of matter dilute independently and standard relationships between the number densities and the temperature ${ }^{4}$ hold. To study alternative

${ }^{3}$ It is expected that the next generation of CMB experiments [44-46] will bring $\Delta n_{s}$ at the $1-\sigma$ confidence level down to 0.002; an improvement by a factor of 3 in comparison with Planck 2015 [1]. The sensitivity for $r$ will come down to $10^{-3}$.

${ }^{4}$ We will find that these relations do not hold in the Einstein frame when we discuss scalars which have nontrivial couplings to the matter fields. 
scenarios for dark matter production, the authors of [26] considered cosmologies where the scalar energy density redshifts as

$$
\rho_{\phi} \propto a^{-(4+n)}, \quad \text { with } n>0 .
$$

The dilution can be expressed in terms of the temperature by making use of conservation of entropy

$$
\rho_{\phi}(T)=\rho_{\phi}\left(T_{r}\right)\left(\frac{g_{s}(T)}{g_{s}\left(T_{r}\right)}\right)^{(4+n) / 3}\left(\frac{T}{T_{r}}\right)^{(4+n)},
$$

where $g_{s}(T)$ is the effective number of d.o.f. associated with the entropy density at temperature $T$, and $T_{r}$ is a reference temperature. The reference temperature is chosen such that the energy density of the scalar and radiation are equal at $T=T_{r}$. With this, the deviation from standard cosmology is completely characterized by the two parameters $\left(n, T_{r}\right)$.

The total energy density can then be written as

$$
\begin{aligned}
\rho(T) & =\rho_{\mathrm{rad}}(T)+\rho_{\phi}(T) \\
& =\rho_{\mathrm{rad}}(T)\left[1+\frac{g\left(T_{r}\right)}{g(T)}\left(\frac{g_{s}(T)}{g_{s}\left(T_{r}\right)}\right)^{(4+n) / 3}\left(\frac{T}{T_{r}}\right)^{n}\right],
\end{aligned}
$$

where $g(T)$ is the number of d.o.f. associated with the energy density of the radiation at temperature $T$. The successes of BBN require that $T_{r}>(15.4)^{1 / n} \mathrm{MeV}$ [26]. Typical values for $T_{r}$ considered in [26] are of the order of $\mathrm{GeV}$. The relentless phenomenon occurs for $n \geq 2$ and $n \geq 4$ for $s$-wave and $p$-wave annihilation, respectively [26]. We will use (4) in Sec. III to calculate the modification to the number of $e$-folds $N_{k}$ due to the pre-BBN nonstandard cosmological evolution described above.

\section{B. Scalar-tensor theories}

In this section, we describe the postinflationary histories associated with a class of scalar-tensor theories, which are motivated from models with extra dimensions. It was shown in [47] that the most general physically consistent relation between two metrics $g_{\mu \nu}$ and $\tilde{g}_{\mu \nu}$ related by a single scalar field $\phi$ includes a conformal $C(\phi)$ as well as a disformal relation $D(\phi)$ as

$$
\tilde{g}_{\mu \nu}=C(\phi) g_{\mu \nu}+D(\phi) \partial_{\mu} \phi \partial_{\nu} \phi .
$$

The presence of the scalar modifies the expansion rate felt by any matter coupled to the "disformal" metric $\tilde{g}_{\mu \nu}$, with interesting implications. The effect of this modified expansion rate for the thermal production of dark matter was recently studied in $[40,41]$. We refer the reader to the literature $[40,41]$ for details of the general setup and derivations.

In these models, the total energy momentum tensor in the Einstein frame, which is defined with respect to the metric $g_{\mu \nu}$, is conserved, $\nabla^{\mu} T_{\mu \nu}^{\mathrm{tot}}=\nabla^{\mu}\left(T_{\mu \nu}^{\phi}+\sum_{i} T_{\mu \nu}^{i}\right)=0$, but the individual fluids are not, that is $\nabla^{\mu} T_{\mu \nu}^{\phi}=-\sum_{i} \nabla^{\mu} T_{\mu \nu}^{i}$. On the other hand, the energy momentum tensor in the Jordan or disformal frame is conserved, $\tilde{\nabla}^{\mu} \tilde{T}_{\mu \nu}=0$, where $\tilde{\nabla}_{\mu}$ is the covariant derivative computed with respect to $\tilde{g}_{\mu \nu}$ and $\tilde{T}_{\mu \nu}$ is the energy momentum tensor computed with respect to the disformal metric $\tilde{g}_{\mu \nu}$. The energy densities and pressures in the two frames can be shown to be given by $[40,41]$

$$
\tilde{\rho}^{i}=C^{-2} \gamma^{-1} \rho^{i}, \quad \tilde{P}^{i}=C^{-2} \gamma P^{i},
$$

where $\gamma=\left(1+\frac{D}{C}(\partial \phi)^{2}\right)^{-1 / 2}$ and the equations of state in both frames are related by $\tilde{\omega}^{i}=\omega^{i} \gamma^{2}$. In the pure conformal case, $\gamma=1$, and thus, $\tilde{\omega}=\omega$. Finally, the Jordan and Einstein frame scale factors are related by

$$
\tilde{a}=C^{1 / 2} a .
$$

The functions $C$ and $D$ can in principle take any form so long as they satisfy the causality constraint $C(\phi)>0$, $C(\phi)+2 X D(\phi)>0$, where $2 X=(\partial \phi)^{2}$. Note also that in our conventions, $C(\phi)$ is dimensionless, while $D(\phi)$ has mass dimension -4. In phenomenological studies, this is the only requirement on $C, D$ and the scalar field takes the standard action of a canonically normalized field. On the other hand, in D-brane scalartensor theories, the scalar action takes the DBI form, $\mathcal{L}=M^{4} C(\phi)^{2} \sqrt{1+2 X D(\phi) / C(\phi)}+V(\phi)$, and the functions $C, D$ are related as $M^{4} C D=1$. Moreover, $M$ is given by the tension of the D-brane, and thus to the string scale (see [41] and below). Finally, in this setup, the inflaton $\varphi$ may, or not, be disformally coupled to the scalar $\phi$, but it will anyhow have some coupling and will thus decay into both $\phi$ and the matter fields coupled to it.

\section{E-FOLDINGS BETWEEN HORIZON EXIT OF CMB MODES AND THE END OF INFLATION}

As discussed in the Introduction, the number of $e$-foldings of the Universe between the horizon exit of the CMB modes and the end of inflation $\left(N_{k}\right)$ is a central input for making predictions for the inhomogeneities in the CMB. The quantity is sensitive to the postinflationary history of the Universe and hence is expected to be altered with any modification of the pre-BBN history of the Universe. In this section, we compute $N_{k}$ for the theories described in Sec. II. In spirit, the computations follow the standard procedure to determine $N_{k}$ (see, e.g., [48] for a recent discussion), but the modified postinflationary histories in these theories lead to nonstandard equations for $N_{k}$.

\section{A. $N_{k}$ for decoupled scalars}

We start from the condition for horizon exit of modes during the inflationary epoch, $k=a_{k} H_{k}$. Where $k$ is the 
comoving wave number of the mode, $a_{k}$ and $H_{k}$ are the scale factor and Hubble constant at the time of horizon exit of the mode. We write the above as

$$
\frac{k}{a_{0}}=\frac{a_{k}}{a_{\mathrm{end}}} \cdot \frac{a_{\mathrm{end}}}{a_{\mathrm{re}}} \cdot \frac{a_{\mathrm{re}}}{a_{0}} \cdot H_{k},
$$

where $a_{\text {end }}, a_{\mathrm{re}}$ are the scale factors at the end of inflation and end of the reheating epoch. $a_{0}$ is the scale factor today. We follow the convention of tracking the evolution in terms of the number of $e$-foldings, (8) then reads

$$
\ln \left(\frac{k}{a_{0}}\right)=-N_{k}-N_{\text {re }}+\ln \left(\frac{a_{\mathrm{re}}}{a_{0}}\right)+\ln H_{k} .
$$

Note that if the entire postinflationary history of the Universe is known then $N_{\text {re }}$ and $\ln \left(a_{\text {re }} / a_{0}\right)$, can be determined by plugging in the values of the scale factor at specific points of the evolution. The Hubble constant at the time of horizon exit is determined in terms of the strength of scalar perturbations

$$
H_{k}^{2}=\frac{\rho_{k}}{3 M_{\mathrm{pl}}^{2}}=\frac{\pi^{2}}{2} M_{\mathrm{pl}}^{2} A_{s} r
$$

where $A_{s}$ is the primordial scalar amplitude and $r$ the tensor to scalar ratio. Thus, the last term in (9) is known modulo the value of $r$; the dependence on $r$ is logarithmic and can be estimated without much uncertainly given a model of inflation. Thus, given a postinflationary history, (9) essentially determines $N_{k}$. While it is a perfectly valid approach to determine $N_{k}$ from (9), it is possible to proceed further generally and determine what features of the postinflationary history have an effect on $N_{k}$. To do this, we begin by a phenomenological parametrization of the reheating epoch, taking the average equation of state during epoch to be $w_{\text {re }}$. We then have

$$
N_{\text {re }}=\ln \left(\frac{a_{\text {re }}}{a_{\text {end }}}\right)=\frac{1}{3\left(1+w_{\text {re }}\right)} \ln \left(\frac{\rho_{\text {end }}}{\rho_{\text {re }}}\right),
$$

where $\rho_{\text {end }}$ is the energy density of the Universe at the end of inflation and $\rho_{\text {re }}$ is the energy density at the end of reheating. We simplify the above by writing

$$
\ln \left(\rho_{\text {end }}\right)=\ln \left(\frac{\rho_{\text {end }}}{\rho_{k}}\right)+\ln \left(\rho_{k}\right) .
$$

The first term involves the ratio of the energy densities at horizon exit and the end of inflation, it can be computed explicitly given the inflationary potential. It typically is small as the energy density remains a constant during the inflationary epoch. The second term is related to the primordial scalar amplitude and $r$ (10). The right-hand side of (11) also involves the energy density at the end of the reheating epoch $\left(\rho_{\text {re }}\right)$. We write this as

$$
\rho_{\mathrm{re}}=\frac{\pi^{2}}{30} g_{\mathrm{re}} T_{\mathrm{re}}^{4} \cdot \eta
$$

where $g_{\mathrm{re}}$ are the effective number of d.o.f. at the end of reheating and $T_{\text {re }}$ the reheat temperature of the Standard Model d.o.f. Usually, the Einstein frame energy density at the end of the reheating epoch is equated to the energy density of the radiation. As discussed in Sec. II, in the theories with a decoupled scalar proposed in [26], the decoupled scalar also carries a fraction of the energy density - we incorporate this in (13) by including the factor $\eta$ (which is equal to the ratio of the total energy density and the energy density of the radiation at the end of reheating). Furthermore, we assume that there is no production of entropy ${ }^{5}$ after the reheating epoch-entropy conservation can then be used to relate the reheat temperature to the temperature of the $\mathrm{CMB}$

$$
T_{\mathrm{re}}=\sqrt{\frac{43}{11 g_{\mathrm{s}, \mathrm{re}}}}\left(\frac{a_{0}}{a_{\mathrm{re}}}\right) T_{0} .
$$

Combining Eqs. (11)-(14), we obtain

$$
\begin{aligned}
\frac{3}{4}\left(1+3 w_{\mathrm{re}}\right) N_{\mathrm{re}}= & \frac{1}{4} \ln \rho_{k}-\ln \left(\frac{a_{0} T_{0}}{a_{\mathrm{re}}}\right)+\frac{1}{4} \ln \left(\frac{\rho_{\mathrm{end}}}{\rho_{k}}\right) \\
& +\beta-\frac{1}{4} \ln \eta,
\end{aligned}
$$

where

$$
\beta=-\frac{1}{4} \ln \left(\frac{\pi^{2}}{30} g_{\mathrm{re}}\right)-\frac{1}{3} \ln \left(\frac{43}{11 g_{\mathrm{s}, \mathrm{re}}}\right) .
$$

Finally, we subtract (15) from (9) to eliminate the dependence on $a_{\mathrm{re}} / a_{0}$. This yields an equation for $N_{k}$,

$$
\begin{aligned}
N_{k}= & -\frac{1}{4}\left(1-3 w_{\text {re }}\right) N_{\text {re }}+\ln H_{k}-\frac{1}{4} \ln \rho_{k} \\
& -\ln \left(\frac{k}{a_{0} T_{0}}\right)-\beta+\frac{1}{4} \ln \left(\frac{\rho_{k}}{\rho_{\text {end }}}\right)+\frac{1}{4} \ln \eta .
\end{aligned}
$$

Let us discuss each term in the right-hand side. The first term depends on the parametrization of reheating $\left(N_{\mathrm{re}}, w_{\mathrm{re}}\right)$. Given the usual uncertainties associated with it, this term is the major source of the uncertainty in $N_{k}$. The second and third terms are determined in terms $A_{s}$ and $r$ (10). In the fourth term, $k / a_{0}$ is the pivot scale for CMB observations and $T_{0}=2.73 \mathrm{~K}$. The fifth term depends on the number of effective d.o.f. at the time of reheating. $\beta \propto \ln \left(g_{\mathrm{re}}^{1 / 4} / g_{\mathrm{s}, \mathrm{re}}^{1 / 3}\right)$; hence, it gives a small contribution. The sixth term involves

\footnotetext{
${ }^{5}$ Entropy production can arise from modulus domination in the postinflationary history [49-51]; the effect of this on inflationary predictions has been analyzed in [52-55].
} 


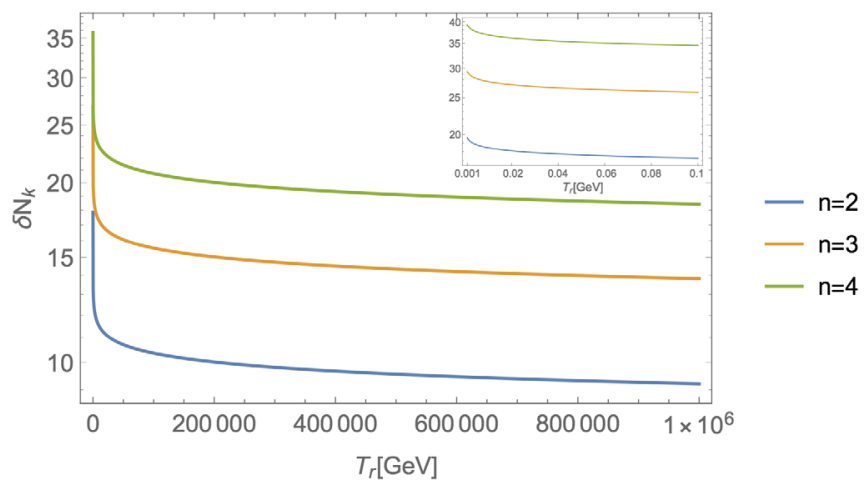

FIG. 1. $\delta N_{k}$ as a function of $T_{r}$, for different values of $n$ with $T_{\text {re }}=10^{14} \mathrm{GeV}$.

the ratio of the energy densities at the time of horizon exit and the end of inflation. It is typically small as the energy density does not change much during inflation. The last term involves the parameter $\eta$ introduced in (13). In conventional cosmological scenarios, this term vanishes as the entire energy density of the Universe is in the form of radiation after reheating. Plugging in the numerical factors, we find ${ }^{6}$

$$
N_{k} \approx 57-\frac{1}{4}\left(1-3 w_{\mathrm{re}}\right) N_{\mathrm{re}}+\frac{1}{4} \ln r+\frac{1}{4} \ln \eta .
$$

As discussed above, in conventional cosmological scenarios $\eta=1$. The term involving the reheating parameters is typically negative, simulations of various scenarios for reheating give $w_{\mathrm{re}}<1 / 3$ (see, e.g., [42,43]). The term involving $r$ is manifestly negative. Given this, Eq. (18) gives motivation for the usual range of 50 to 60 for $N_{k}$ in conventional cosmologies, (i.e., $\eta=1$ ). We can read off the expression for $\eta$ (in terms of $n$ and $T_{r}$ ) from the expression for the total energy in the postinflationary epoch. ${ }^{7}$ Equation (4) gives

$$
\eta \approx \frac{g\left(T_{r}\right)}{g\left(T_{\mathrm{re}}\right)}\left(\frac{g_{s}\left(T_{\mathrm{re}}\right)}{g_{s}\left(T_{r}\right)}\right)^{(4+n) / 3}\left(\frac{T_{\mathrm{re}}}{T_{r}}\right)^{n} .
$$

Recall that the $\eta$ contribution to $N_{k}$ is $\delta N_{k} \equiv 1 / 4 \ln \eta$ (17).

As was shown in [26], the largest effect in the dark matter decoupling is obtained for smaller $T_{r}$ 's so long as the BBN predictions are not affected, which gave $T_{r} \simeq 10 \mathrm{MeV}$. From (19), we see that the largest effect in $\delta N_{k}$ for a given reheating temperature will precisely be given for the smallest $T_{r}$ 's as shown in Fig. 1.

Taking, for example, a reheating temperature of $T_{\text {re }}=10^{14} \mathrm{GeV}$, for $n=2$ and $T_{r}=1 \mathrm{GeV}$, one obtains $\delta N_{k} \approx 16$. For $n=2, T_{r}=10^{6} \mathrm{GeV}, \delta N_{k} \approx 9$. Since $n$

\footnotetext{
${ }^{6}$ We take $g_{\text {re }} \approx g_{\mathrm{s} \text {, re }} \approx 100$ and $\rho_{\text {end }} / \rho_{k} \approx 1$.

${ }^{7}$ Assuming $\frac{g\left(T_{r}\right)}{g\left(T_{\mathrm{re}}\right)}\left(\frac{g_{s}\left(T_{\mathrm{re}}\right)}{g_{s}\left(T_{r}\right)}\right)^{(4+n) / 3} \sim \mathcal{O}(1)$. Note that in general, this factor is greater than one; hence, when relevant, it will lead to a further enhancement of the effect.
}

appears in the exponent in (19), higher values of $n$ can lead to much larger shifts in $N_{k}$. For $n=4$ (recall that this is the critical value of $n$ for relentless dark matter if dark matter annihilation is a $p$-wave process [26]) with $T_{r}=1 \mathrm{GeV}$ yields $\delta N_{k} \approx 32$, while $T_{r}=10^{6} \mathrm{GeV}$ yields $\delta N_{k} \approx 18$. Note that since $T_{\mathrm{re}} \gg T_{r}$, the change in $\delta N_{k}$ is larger for smaller values of $T_{r}$ (see inset in Fig. 1). For example, for a factor $m \sim \mathcal{O}(1)$ change in $T_{\mathrm{r}}$, the change in $\delta N_{k} \sim \frac{n}{4} \log m$, which gives an $\mathcal{O}(1)$ change (see inset in Fig. 1).

We will discuss the implications of our results for confronting inflationary models with data in Sec. IV; here we would like to emphasize that shifts in the value of $N_{k}$ obtained above can have a significant effect on the $\left(n_{s}, r\right)$ predictions of any inflationary model. Also, note that since $\eta$ is the ratio of the total energy density and the energy density in radiation at the end of reheating, $\eta>1$; hence, the effect of the last term in (18) is to increase $N_{k}$. The positivity of the shift is natural as in the epoch in which the scalar dominates the energy density in the postinflationary history, the energy density dilutes as $a^{-(4+n)}$, i.e., faster than radiation. This is similar to a kination phase [56-59], which is known to make a positive contribution to $N_{k}$ (see, e.g., [60] for a recent discussion). Of course, the key difference is that this phase occurs after reheating.

\section{B. $N_{k}$ for coupled scalars}

In this section, we derive the expression for the number of $e$-foldings between horizon exit of CMB modes and the end of inflation $\left(N_{k}\right)$ for the coupled case where the scalar arises from a scalar-tensor theory and thus couples both conformal and disformally to matter. The crucial difference from the discussion in the previous section is that the standard relations for energy and entropy conservation now hold in the Jordan frame. ${ }^{8}$ The analogous expression for (9) in the present case is given by

$$
\ln \left(\frac{k}{a_{0}}\right)=-N_{k}-\tilde{N}_{\text {re }}+\ln \left(\frac{\tilde{a}_{\mathrm{re}}}{a_{0}}\right)+\ln H_{k},
$$

where

$$
\tilde{N}_{\text {re }}=\ln \frac{\tilde{a}_{\text {re }}}{\tilde{a}_{\text {end }}}=N_{\text {re }}+\frac{1}{2} \ln \frac{C_{\text {re }}}{C_{\text {end }}},
$$

and since the scalar is not active at the end of inflation, we can take $\tilde{a}_{\text {end }}=a_{\text {end }}$ (that is, $C_{\text {end }}=1$ ). Moreover, since standard GR evolution must be reached at the onset of BBN to avoid spoiling its predictions, we also have $\tilde{a}_{0}=a_{0}$. Now, using energy conservation in the Jordan frame, we can write

\footnotetext{
${ }^{8}$ As mentioned earlier, we shall denote all Jordan frame quantities by a tilde superscript to distinguish them from Einstein frame quantities.
} 
$\tilde{N}_{\text {re }}=\ln \left(\frac{\tilde{a}_{\text {re }}}{a_{\text {end }}}\right)=\frac{1}{3\left(1+w_{\text {end }}\right)} \ln \rho_{\text {end }}-\frac{1}{3\left(1+\tilde{w}_{\text {re }}\right)} \ln \tilde{\rho}_{\text {re }}$.

We gave an expression for $\rho_{\text {end }}$ in (12) in terms of $\rho_{k}$. On the other hand, in the Jordan frame, we have $[40,41]$

$$
\tilde{\rho}_{\mathrm{re}}=\frac{\pi^{2}}{30} g_{\mathrm{re}} \tilde{T}_{\mathrm{re}}^{4}
$$

where $g_{\mathrm{re}}$ is the effective number of d.o.f. (which in principle depend on $\tilde{T}$ ) at the end of reheating and $\tilde{T}_{\text {re }}$ is the Jordan frame reheat temperature. Furthermore, assuming as before that there is no production of entropy after the reheating epoch-entropy conservation in the Jordan frame can be used to relate the reheat temperature to the temperature of the $\mathrm{CMB}$,

$$
\tilde{T}_{\text {re }}=\left(\frac{43}{11 g_{\mathrm{s}, \mathrm{re}}}\right)^{1 / 3}\left(\frac{a_{0}}{\tilde{a}_{\mathrm{re}}}\right) \tilde{T}_{0}
$$

Using these equations, we arrive at an expression for $N_{k}$ in terms of $\tilde{N}_{\text {re }}$,

$$
\begin{aligned}
N_{k}= & -\frac{1}{4}\left(1-3 \tilde{\omega}_{\text {re }}\right) \tilde{N}_{\text {re }}+\ln H_{k}-\beta-\ln \left(\frac{k}{a_{0} T_{0}}\right) \\
& -\frac{\left(1+\tilde{\omega}_{\text {re }}\right)}{4\left(1+\omega_{\text {end }}\right)}\left[\ln \left(\frac{\rho_{\text {end }}}{\rho_{k}}\right)+\ln \rho_{k}\right] .
\end{aligned}
$$

Using (21), and considering that $\tilde{\omega}_{\text {re }} \sim \omega_{\text {end }}$, we arrive at

$$
\begin{aligned}
N_{k}= & -\frac{1}{4}\left(1-3 \tilde{\omega}_{\mathrm{re}}\right) N_{\mathrm{re}}+\ln H_{k}-\beta-\ln \left(\frac{k}{a_{0} T_{0}}\right) \\
& -\frac{1}{4} \ln \left(\frac{\rho_{\text {end }}}{\rho_{k}}\right)+\frac{1}{4} \ln \rho_{k}-\frac{1}{8}\left(1-3 \tilde{\omega}_{\mathrm{re}}\right) \ln C_{\mathrm{re}} .
\end{aligned}
$$

In general $C(\phi)$ depends on time (or temperature). However, as studied in [41], a pure disformal term with $C=C_{0}=$ const gives a nontrivial enhancement of the expansion rate and thus an earlier dark matter freeze-out than in the standard case. This causes a relentlessness effect, similar to the decoupled case discussed in the previous section. Therefore a constant $C_{0}$ is the most interesting example. Furthermore, we can also assume a Jordan frame equation of state $\tilde{\omega}_{r e}$ to be roughly constant.

In the case of a D-brane scalar tensor model [41], the disformal and conformal functions arise from the DiracBorn-Infeld (DBI) action as discussed above. The scale $M$ introduced there is related to the tension of a D3-brane, which is given by the string scale as $M=M_{s}\left(2 \pi g_{s}^{-1}\right)^{1 / 4}$. Defining the scale $\tilde{M}=M C_{0}^{1 / 4}$, the interesting cases for the modified dark matter thermal scenarios occurred for $\tilde{M} \in$ $(10-300) \mathrm{GeV}$ in [41]. Considering a typical string scale, which arises in string theory models of inflation, $M_{s} \sim$ $\left(10^{14}-10^{16}\right) \mathrm{GeV}$ (with $g_{s} \sim 0.1$ ) allows us to find the value of $C_{0}$ and thus the modification to $N_{k}$. The net effect is a positive modification to $N_{k}$, hence increasing the required number of $e$-folds by about $\delta N_{k} \sim 15$ (for $\tilde{\omega}_{\text {re }} \sim 0$ ), and thus similar to the decoupled example with, e.g., $n=2$, $T_{r}=1 \mathrm{GeV}$, and $T_{\mathrm{re}}=10^{14} \mathrm{GeV}$. In terms of a D3-brane picture, this can be interpreted as the D-brane moving at the tip of a warped throat.

The case $C_{0}=1$, on the other hand, would correspond to an unwarped geometry. In this case, $\tilde{M}=M$, and therefore an effect in the thermal dark matter production is tied to the string scale. In this case, the relevant values of $M$ for a relentless dark matter effect require extremely low string scales [41]. In the phenomenological case, on the other hand, the values of $C_{0}$ and $D_{0}$ are in principle unconstrained and therefore can again give large positive corrections to $\delta N_{k}$.

\section{IMPLICATIONS FOR INFLATIONARY PREDICTIONS}

In this section, we discuss the implications of the shifts in $N_{k}$ derived in Sec. III for the predictions of inflationary models in the $\left(n_{s}, r\right)$ plane. Inflationary models fall into universality classes characterized by the relationship between $n_{s}, r$, and $N_{k}$ [61]. It is natural to use this classification to study how the shift in $N_{k}$ can affect various models. Class I models obey the relations

$$
n_{s} \simeq 1+\frac{2 \lambda}{N_{k}}, \quad r \sim \frac{1}{N_{k}^{-2 \lambda}} \lambda<-\frac{1}{2},
$$

where $\lambda$ is a constant which depends on the inflationary model. Note that an increase $N_{k}$ implies that models with higher values of $\eta$ are preferred (similarly higher values of $N_{k}$ imply higher values of $\eta$ ). The prototypical models for class I are hilltop [62] and Starobinsky [63]. Amongst the models obtained from string theory, it includes the fibre inflation model [64-66]. The Starobinsky model has

$$
n_{s} \simeq 1-\frac{2}{N}, \quad r \simeq \frac{12}{N^{2}},
$$

which corresponds to $\lambda=-1$. For these models, lowering of $N_{k}$ (as found in [52]) can be interesting as this pushes up the value of $r$; the increase in $N_{k}$ found for the models makes the size of $r$ even smaller.

Class II models are characterized by

$$
n_{s} \simeq 1-\frac{2 \lambda+1}{N}, \quad r \simeq \frac{16 \lambda}{N},
$$

where again $\lambda$ is a constant depending on the inflationary model. These are large field models. The prototypes for these are the monomial potentials: $V(\varphi)=\frac{1}{2} m^{4-\alpha} \varphi^{\alpha}$ ( $\alpha=2$ corresponds to the famous $m^{2} \varphi^{2}$ model [67-69], 


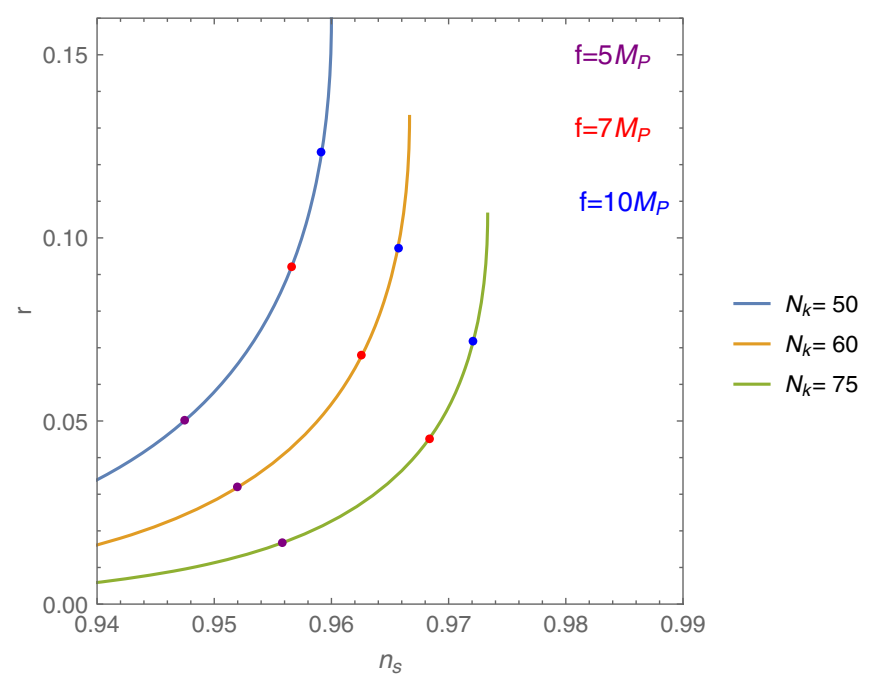

FIG. 2. Predictions of natural inflation in the $\left(n_{s}, r\right)$ plane.

$\alpha=2 / 3,1$ arise in the axion monodromy examples $[70,71])$. The predictions in the $\left(n_{s}, r\right)$ plane are

$$
n_{s}=1-\frac{\alpha+2}{2 N} ; \quad r=\frac{4 \alpha}{N} .
$$

For the $m^{2} \varphi^{2}$ model, $N_{k}$ in the range of 50 to 60 gives $n_{s}$ in the range 0.960 to 0.966 and $r$ in the range 0.13 to 0.16 . Recall that PLANCK 2015 (TT + TE + EE) + low P + lensing for the $\Lambda \mathrm{CDM}+r$ model gives $n_{s}=0.9688 \pm$ 0.0061 and $r<0.114$ at $1-\sigma$ [1]). The major tension with data arises from the high value of the prediction for $r$. This can be ameliorated with an increase in the value in $N_{k}$. For example, $N_{k}=72$ gives $r=0.11$ and $n_{s}=0.972$, which is within the Planck $20151-\sigma$ range for $n_{s}$ and $r$. In scenarios for which the reheating epoch is not extended, this corresponds to $\delta N_{k} \approx+16$. Such a shift is possible for a large range of the model parameters both the decoupled and coupled scalar cases, particularly in the case of relentless dark matter and the D-brane scalar-tensor model [41]. We note that although the shift brings both $n_{s}$ and $r$ within their $1-\sigma$ values, the model only enters the $2-\sigma$ region in the marginalized joint distribution in the $\left(n_{s}, r\right)$ plane. ${ }^{9}$ But overall the shift in the predictions is in the direction favored by the present data. For the linear potential $(\alpha=1), N_{k}$ in the range of 50 to 60 gives $n_{s}$ in the range 0.980 to 0.983 and $r$ in the range 0.080 to 0.066 . In this case, the prediction for $n_{s}$ is on the higher side and increase in $N_{k}$ further increases the tension in the $n_{s}$ prediction. The situation for $\alpha=2 / 3$ is similar.

Another interesting class of models is that of natural inflation [72]. In this case, the dependence on $N$ of the cosmological parameters $\left(n_{s}, r\right)$ is nonperturbative with

\footnotetext{
${ }^{9}$ With the Planck 2015 data, it impossible for the model to enter $1-\sigma$ region in the joint distribution; for the model to predict $r$ values in the necessary range requires $N>100$, but in this regime, the predictions for $n_{s}$ are incompatible with the data.
}

$n_{s}=1+\frac{1}{f^{2}} \frac{\left(e^{N_{k} / f^{2}}+1\right)}{\left(e^{N_{k} / f^{2}}-1\right)}, \quad r=\frac{8}{f^{2}} \frac{1}{e^{N_{k} / f^{2}}-1}$,

where $f$ is the axion decay constant (in Planck units). Therefore, a larger value of $N_{k}$ can bring this model back into the allowed region of the parameters. For example, for $f=7 M_{P}, N_{k}=60$, we have $\left(n_{s}, r\right)=(0.962,0.07)$, while $f=7 M_{P}, N_{k}=75$ gives $\left(n_{s}, r\right)=(0.968,0.05)$. We show the model predictions as a function of $N_{k}$ in Fig. 2.

\section{CONCLUSIONS}

Scalar fields are ubiquitous in theories beyond standard models of cosmology and particle physics. During the early pre-BNN cosmological history, these can appear as an extra species, decoupled from matter, or in scalar-tensor theories, where the gravitational interaction is mediated by both the metric and scalar field, coupling to matter conformal and disformally.

These scalars evolve during the pre-BNN epoch, giving rise to interesting implications for dark matter relic abundances [26,39-41]. The modified postinflationary history implies a shift in the number of $e$-foldings between the horizon exit of CMB modes and the end of inflation, $N_{k}$. At the same time, this implies a change in the inflationary parameters $\left(n_{s}, r\right)$. We have computed this shift for theories with decoupled scalars and scalar-tensor theories and examined the effect that this can have on the predictions in the $\left(n_{s}, r\right)$ plane for inflationary models. In particular, we considered the models discussed recently in [26] when an extra scalar species modifies the dark matter (DM) relic abundance and the disformal models arising from D-brane scalar-tensor theories discussed in [40,41], where the scalar couples conformal and disformally to matter, modifying the expansion rate and thus the DM relic abundances.

For decoupled scalars, we found that the shift in $N_{k}$ is positive definite. The case of relentless DM gives the largest effect with $\delta N_{k} \approx 15$ or larger, with a reheating temperature of around $T_{\text {re }} \sim 10^{14} \mathrm{GeV}$ (see Sec. III A). For the scalar-tensor case, we derived $\delta N_{k}$ for a disformal enhancement of the expansion rate as studied in [41]. This corresponds to constant conformal and disformal functions $C=C_{0}, D_{0}=\tilde{M}^{-4}=\left(C_{0} M^{4}\right)^{-1}$. For the values of $\tilde{M}$ and $C_{0}$ relevant for the modifications of the expansion rate and thus relic DM abundance [41], we found a large positive effect with $\delta N_{k} \approx 15$ (see Sec. III B).

We used this to study the implications for the predictions of $\left(n_{s}, r\right)$ for some interesting inflationary models. For example, the increase in $N_{k}$ implies the possibility of a decrease in the prediction for $r$ for the $m^{2} \varphi^{2}$ model, which reduces the tension with data. The change in $N_{k}$ has also an important effect for natural inflation, by decreasing $r$ and moving $n_{s}$ towards the allowed values of Planck. Analysis in the spirit of this work will become more and more important as we probe the CMB even more minutely. 


\section{ACKNOWLEDGMENTS}

We would like to thank the organisers of the workshop on Postinflationary String Cosmology, Bologna 2017 for the simulating environment which led this collaboration. A. M. is partially supported by a Ramanujan Fellowship, DST, Government of India. I. Z. is partially supported by STFC Grant No. ST/P00055X/1.

[1] P. A. R. Ade et al. (Planck Collaboration), Planck 2015 results. XIII. Cosmological parameters, Astron. Astrophys. 594, A13 (2016).

[2] A. Albert et al. (DES, Fermi-LAT Collaboration), Searching for dark matter annihilation in recently discovered Milky Way satellites with Fermi-LAT, Astrophys. J. 834, 110 (2017).

[3] A. U. Abeysekara et al. (HAWC Collaboration), Sensitivity of HAWC to high-mass dark matter annihilations, Phys. Rev. D 90, 122002 (2014).

[4] J. Carr et al. (CTA Collaboration), Prospects for indirect dark matter searches with the Cherenkov Telescope Array (CTA), Proc. Sci., ICRC2015 (2016) 1203.

[5] Dark Matter Hub, https://www.interactions.org/hub/darkmatter-hub.

[6] J. D. Barrow, Massive particles as a probe of the early Universe, Nucl. Phys. B208, 501 (1982).

[7] J. McDonald, WIMP densities in decaying particle dominated cosmology, Phys. Rev. D 43, 1063 (1991).

[8] M. Kamionkowski and M. S. Turner, Thermal relics: Do we know their abundances?, Phys. Rev. D 42, 3310 (1990).

[9] T. Moroi, M. Yamaguchi, and T. Yanagida, On the solution to the Polonyi problem with $0(10-\mathrm{TeV})$ gravitino mass in supergravity, Phys. Lett. B 342, 105 (1995).

[10] D. J. H. Chung, E. W. Kolb, and A. Riotto, Production of massive particles during reheating, Phys. Rev. D 60, 063504 (1999).

[11] M. Hashimoto, K. I. Izawa, M. Yamaguchi, and T. Yanagida, Gravitino overproduction through moduli decay, Prog. Theor. Phys. 100, 395 (1998).

[12] T. Moroi and L. Randall, Wino cold dark matter from anomaly mediated SUSY breaking, Nucl. Phys. B570, 455 (2000).

[13] G. F. Giudice, E. W. Kolb, and A. Riotto, Largest temperature of the radiation era and its cosmological implications, Phys. Rev. D 64, 023508 (2001).

[14] R. Allahverdi and M. Drees, Production of Massive Stable Particles in Inflaton Decay, Phys. Rev. Lett. 89, 091302 (2002).

[15] M. Endo, K. Hamaguchi, and F. Takahashi, Moduli-Induced Gravitino Problem, Phys. Rev. Lett. 96, 211301 (2006).

[16] S. Nakamura and M. Yamaguchi, Gravitino production from heavy moduli decay and cosmological moduli problem revived, Phys. Lett. B 638, 389 (2006).

[17] B. S. Acharya, P. Kumar, K. Bobkov, G. Kane, J. Shao, and S. Watson, Non-thermal dark matter and the moduli problem in string frameworks, J. High Energy Phys. 06 (2008) 064.
[18] R. Allahverdi, B. Dutta, and K. Sinha, Non-thermal Higgsino dark matter: Cosmological motivations and implications for a $125 \mathrm{GeV}$ Higgs, Phys. Rev. D 86, 095016 (2012).

[19] R. Allahverdi, B. Dutta, R. N. Mohapatra, and K. Sinha, A Supersymmetric Model for Dark Matter and Baryogenesis Motivated by the Recent CDMS Result, Phys. Rev. Lett. 111, 051302 (2013).

[20] R. Allahverdi, M. Cicoli, B. Dutta, and K. Sinha, Nonthermal dark matter in string compactifications, Phys. Rev. D 88, 095015 (2013).

[21] R. T. Co, F. D’Eramo, L. J. Hall, and D. Pappadopulo, Freeze-in dark matter with displaced signatures at colliders, J. Cosmol. Astropart. Phys. 12 (2015) 024.

[22] L. Aparicio, M. Cicoli, B. Dutta, S. Krippendorf, A. Maharana, F. Muia, and F. Quevedo, Non-thermal CMSSM with a 125 GeV Higgs, J. High Energy Phys. 05 (2015) 098.

[23] K. Kannike, A. Racioppi, and M. Raidal, Super-heavy dark matter-Towards predictive scenarios from inflation, Nucl. Phys. B918, 162 (2017).

[24] M. Kawasaki, K. Kohri, and N. Sugiyama, Cosmological Constraints on Late Time Entropy Production, Phys. Rev. Lett. 82, 4168 (1999).

[25] M. Kawasaki, K. Kohri, and N. Sugiyama, MeV scale reheating temperature and thermalization of neutrino background, Phys. Rev. D 62, 023506 (2000).

[26] F. D'Eramo, N. Fernandez, and S. Profumo, When the Universe expands too fast: Relentless dark matter, J. Cosmol. Astropart. Phys. 05 (2017) 012.

[27] R. Catena, N. Fornengo, M. Pato, L. Pieri, and A. Masiero, Thermal relics in modified cosmologies: Bounds on evolution histories of the early Universe and cosmological boosts for PAMELA, Phys. Rev. D 81, 123522 (2010).

[28] G. B. Gelmini, J.-H. Huh, and T. Rehagen, Asymmetric dark matter annihilation as a test of non-standard cosmologies, J. Cosmol. Astropart. Phys. 08 (2013) 003.

[29] T. Rehagen and G. B. Gelmini, Effects of kination and scalar-tensor cosmologies on sterile neutrinos, J. Cosmol. Astropart. Phys. 06 (2014) 044.

[30] S.-z. Wang, H. Iminniyaz, and M. Mamat, Asymmetric dark matter and the scalar-tensor model, Int. J. Mod. Phys. A 31, 1650021 (2016).

[31] A. Lahanas, N. Mavromatos, and D. V. Nanopoulos, Dilaton and off-shell (non-critical string) effects in Boltzmann equation for species abundances, PMC Phys. A 1, 2 (2007).

[32] C. Pallis, Cold dark matter in non-standard cosmologies, PAMELA, ATIC and Fermi LAT, Nucl. Phys. B831, 217 (2010). 
[33] P. Salati, Quintessence and the relic density of neutralinos, Phys. Lett. B 571, 121 (2003).

[34] A. Arbey and F. Mahmoudi, SUSY constraints from relic density: High sensitivity to pre-BBN expansion rate, Phys. Lett. B 669, 46 (2008).

[35] H. Iminniyaz and X. Chen, Relic abundance of asymmetric dark matter in quintessence, Astropart. Phys. 54, 125 (2014).

[36] M. T. Meehan and I. B. Whittingham, Asymmetric dark matter in braneworld cosmology, J. Cosmol. Astropart. Phys. 06 (2014) 018.

[37] M. T. Meehan and I. B. Whittingham, Dark matter relic density in Gauss-Bonnet braneworld cosmology, J. Cosmol. Astropart. Phys. 12 (2014) 034.

[38] M. T. Meehan and I. B. Whittingham, Dark matter relic density in scalar-tensor gravity revisited, J. Cosmol. Astropart. Phys. 12 (2015) 011.

[39] R. Catena, N. Fornengo, A. Masiero, M. Pietroni, and F. Rosati, Dark matter relic abundance and scalar-tensor dark energy, Phys. Rev. D 70, 063519 (2004).

[40] B. Dutta, E. Jimenez, and I. Zavala, Dark matter relics and the expansion rate in scalar-tensor theories, J. Cosmol. Astropart. Phys. 06 (2017) 032.

[41] B. Dutta, E. Jimenez, and I. Zavala, D-brane disformal coupling and thermal dark matter, Phys. Rev. D 96, 103506 (2017).

[42] R. Allahverdi, R. Brandenberger, F.-Y. Cyr-Racine, and A. Mazumdar, Reheating in inflationary cosmology: Theory and applications, Annu. Rev. Nucl. Part. Sci. 60, 27 (2010).

[43] D. I. Podolsky, G. N. Felder, L. Kofman, and M. Peloso, Equation of state and beginning of thermalization after preheating, Phys. Rev. D 73, 023501 (2006).

[44] K. N. Abazajian et al. (CMB-S4 Collaboration), CMB-S4 Science Book First Edition, arXiv:1610.02743.

[45] T. Matsumura et al., Mission design of LiteBIRD, J. Low Temp. Phys. 176, 733 (2014).

[46] F. Finelli et al. (CORE Collaboration), Exploring cosmic origins with CORE: Inflation, J. Cosmol. Astropart. Phys. 04 (2018) 016.

[47] J. D. Bekenstein, The relation between physical and gravitational geometry, Phys. Rev. D 48, 3641 (1993).

[48] L. Dai, M. Kamionkowski, and J. Wang, Reheating Constraints to Inflationary Models, Phys. Rev. Lett. 113, 041302 (2014).

[49] G. D. Coughlan, R. Holman, P. Ramond, and G. G. Ross, Supersymmetry and the entropy crisis, Phys. Lett. 140B, 44 (1984).

[50] B. de Carlos, J. A. Casas, F. Quevedo, and E. Roulet, Model independent properties and cosmological implications of the dilaton and moduli sectors of 4-d strings, Phys. Lett. B 318, 447 (1993).

[51] T. Banks, D. B. Kaplan, and A. E. Nelson, Cosmological implications of dynamical supersymmetry breaking, Phys. Rev. D 49, 779 (1994).
[52] K. Dutta and A. Maharana, Inflationary constraints on modulus dominated cosmology, Phys. Rev. D 91, 043503 (2015).

[53] K. Das, K. Dutta, and A. Maharana, Inflationary predictions and moduli masses, Phys. Lett. B 751, 195 (2015).

[54] M. Cicoli, K. Dutta, A. Maharana, and F. Quevedo, Moduli vacuum misalignment and precise predictions in string inflation, J. Cosmol. Astropart. Phys. 08 (2016) 006.

[55] S. Bhattacharya, K. Dutta, M. R. Gangopadhyay, and A. Maharana, Confronting Kähler moduli inflation with $\mathrm{CMB}$ data, arXiv:1711.04807.

[56] B. Spokoiny, Deflationary universe scenario, Phys. Lett. B 315, 40 (1993).

[57] M. Joyce and T. Prokopec, Turning around the sphaleron bound: Electroweak baryogenesis in an alternative postinflationary cosmology, Phys. Rev. D 57, 6022 (1998).

[58] C. Pallis, Quintessential kination and cold dark matter abundance, J. Cosmol. Astropart. Phys. 10 (2005) 015.

[59] D. J. H. Chung, L. L. Everett, and K. T. Matchev, Inflationary cosmology connecting dark energy and dark matter, Phys. Rev. D 76, 103530 (2007).

[60] K. Dimopoulos and C. Owen, Quintessential nflation with $\alpha$-attractors, J. Cosmol. Astropart. Phys. 06 (2017) 027.

[61] D. Roest, Universality classes of inflation, J. Cosmol. Astropart. Phys. 01 (2014) 007.

[62] L. Boubekeur and D. H. Lyth, Hilltop inflation, J. Cosmol. Astropart. Phys. 07 (2005) 010.

[63] A. A. Starobinsky, A new type of isotropic cosmological models without singularity, Phys. Lett. 91B, 99 (1980).

[64] M. Cicoli, C. P. Burgess, and F. Quevedo, Fibre inflation: Observable gravity waves from IIB string compactifications, J. Cosmol. Astropart. Phys. 03 (2009) 013.

[65] C. P. Burgess, M. Cicoli, S. de Alwis, and F. Quevedo, Robust inflation from fibrous strings, J. Cosmol. Astropart. Phys. 05 (2016) 032.

[66] M. Cicoli, D. Ciupke, V. A. Diaz, V. Guidetti, F. Muia, and P. Shukla, Chiral global embedding of fibre inflation models, J. High Energy Phys. 11 (2017) 207.

[67] A. D. Linde, Chaotic inflation, Phys. Lett. 129B, 177 (1983).

[68] V. A. Belinsky, I. M. Khalatnikov, L. P. Grishchuk, and Ya. B. Zeldovich, Inflationary stages in cosmological models with a scalar field, Phys. Lett. 155B, 232 (1985).

[69] T. Piran and R. M. Williams, Inflation in universes with a massive scalar field, Phys. Lett. 163B, 331 (1985).

[70] L. McAllister, E. Silverstein, and A. Westphal, Gravity waves and linear inflation from axion monodromy, Phys. Rev. D 82, 046003 (2010).

[71] E. Silverstein and A. Westphal, Monodromy in the CMB: Gravity waves and string inflation, Phys. Rev. D 78, 106003 (2008).

[72] K. Freese, J. A. Frieman, and A. V. Olinto, Natural Inflation with Pseudo-Nambu-Goldstone Bosons, Phys. Rev. Lett. 65, 3233 (1990). 